Trauma Berufskrankh 2012 - 14[Suppl 2]:197-202 DOI 10.1007/s10039-012-1856-y

Online publiziert: 24. Mai 2012

(c) Springer-Verlag 2012

P.M. Vogt • M. Hiller

Klinik und Poliklinik für Plastische, Hand- und Wiederherstellungschirurgie,

Medizinische Hochschule Hannover

\title{
N.-radialis-Ausfälle im Oberarmbereich
}

\section{Wann muss wie reagiert werden?}

Der Symptomenkomplex der sog. Fallhand als Folge einer Läsion des N. radialis entsteht in unterschiedlicher Ausprägung je nach Höhe der Läsion im Verlauf des Nervs. Betroffene Patienten leiden sehr unter den Folgen und drängen häufig auf eine rasche Operation, von der sie eine komplette Wiederherstellung der Funktion erwarten. Wann und wie man eine isolierte Läsion des N. radialis am

\begin{tabular}{|l|l}
\hline \multicolumn{2}{|l}{ Abkürzungen } \\
\hline$A P L$ & M. abductor pollicis longus \\
\hline$B R$ & M. brachioradialis \\
\hline$C$ & Zervikal \\
\hline$D$ & Digitus \\
\hline$E C R B$ & M. extensor carpi radialis brevis \\
\hline$E C R L$ & M. extensor carpi radialis longus \\
\hline$E C U$ & M. extensor carpi ulnaris \\
\hline$E D C$ & M. extensor digitorum communis \\
\hline$E D$ quinti & M. extensor digiti quinti \\
\hline$E D M$ & M. extensor digiti minimi \\
\hline$E I P$ & M. extensor indicis proprius \\
\hline$E M G$ & Elektromyogramm \\
\hline$E P B$ & M. extensor pollicis brevis \\
\hline$E P L$ & M. extensor pollicis longus \\
\hline$F C R$ & M. flexor carpi radialis \\
\hline$F C U$ & M flexor carpi ulnaris \\
\hline$F D P$ & M. flexor digitorum profundus \\
\hline$F D S$ & M. flexor digitorum superficialis \\
\hline$F P B$ & M. flexor pollicis brevis \\
\hline$F P L$ & M. flexor pollicis longus \\
\hline$K G$ & Krankengymnastik \\
\hline$M P$ & Metakarpophalangeal \\
\hline$M R T$ & Magnetresonanztomographie \\
\hline$P L$ & M. palmaris longus \\
\hline$T h$ & Thorakal \\
\hline & \\
\hline$E D$ &
\end{tabular}

Besten therapiert und welche Erfolgsaussichten bestehen, hängt von

- der Art der Verletzung,

- den Symptomen,

- den Möglichkeiten einer primären Rekonstruktion und

- der seit stattgehabter Verletzung bereits vergangenen Zeit

ab. Das therapeutische Vorgehen sollte daher individuell mit jedem Patienten besprochen und ein Therapieplan festgelegt werden.

Eine frühzeitige Sehnentransposition nur geringen Ausmaßes kann z. B: die Restfunktion deutlich verbessern und Kontrakturen vorbeugen, ist jedoch bei im weiteren Verlauf auftretender Regeneration der Nervenfunktion auch wieder rückgängig zu machen, sodass der Patient durch die frühere Therapie keine Nachteile erleidet. Andererseits kann bei kooperativen Patienten auch zunächst eine ergotherapeutische Behandlung, welche heutzutage vielfältige Möglichkeiten zur akti- ven Beübung mit modernen Zügelsystemen und Nachtlagerungsschienen bietet, erfolgen, um die Zeit bis zur Nervenregeneration, egal ob spontan oder durch eine im weiteren Verlauf durchgeführte Rekonstruktion bei ausbleibender Regeneration, zu überbrücken. Die Ursache der Verletzung ist daher bei der Wahl der Therapie unbedingt zu berücksichtigen.

Eine isolierte Läsion des N. radialis, z. B. im Rahmen einer Stich- oder Schnittverletzung, ist eher selten.

Durch die anatomisch exponierte Lage mit dem Verlauf um den Oberarmknochen ist der N. radialis bei einer Fraktur des Humerus, v. a. bei Schaftfrakturen im distalen Drittel, besonders gefährdet. Die verletzungsbedingte Radialisparese stellt sogar die häufigste traumatische Nervenläsion bei Schädigungen der langen Röhrenknochen dar. Nach sowohl offener als auch geschlossener Oberarmschaftfraktur kommt es laut Shao et al. [33] in 11,8\% der Fälle zu einem teilweisen oder kompletten Ausfall der Nervenfunktion. Da-

\begin{tabular}{|c|c|c|}
\hline \multirow[t]{3}{*}{ Trauma } & \multirow{2}{*}{$\begin{array}{l}\text { Frakturen des Humerus } \\
\text { (v. a. Schaftbrüche) }\end{array}$} & Offene Frakturen \\
\hline & & Geschlossene Frakturen \\
\hline & Weichteilverletzungen & $\begin{array}{l}\text { Stich-, schnitt- und schussverletzungsbedingte } \\
\text { Nervenläsionen }\end{array}$ \\
\hline \multirow[t]{4}{*}{ Druckschädigungen } & \multicolumn{2}{|c|}{ Tumorwachstum am Oberarm } \\
\hline & \multicolumn{2}{|l|}{ Axillare Krücken } \\
\hline & \multicolumn{2}{|c|}{ Lagerungsschäden in Narkose } \\
\hline & \multicolumn{2}{|c|}{ Drogenrausch (Parkbanklähmung unter Alkoholeinfluss) } \\
\hline \multirow[t]{2}{*}{ latrogene Ursachen } & Osteosynthesen & $\begin{array}{l}\text { Insbesondere am Humerusschaft } \\
\text { Metallentfernung } \\
\text { Umstellungsosteotomie }\end{array}$ \\
\hline & \multicolumn{2}{|c|}{ Injektion am Oberarm (Außenseite) } \\
\hline
\end{tabular}




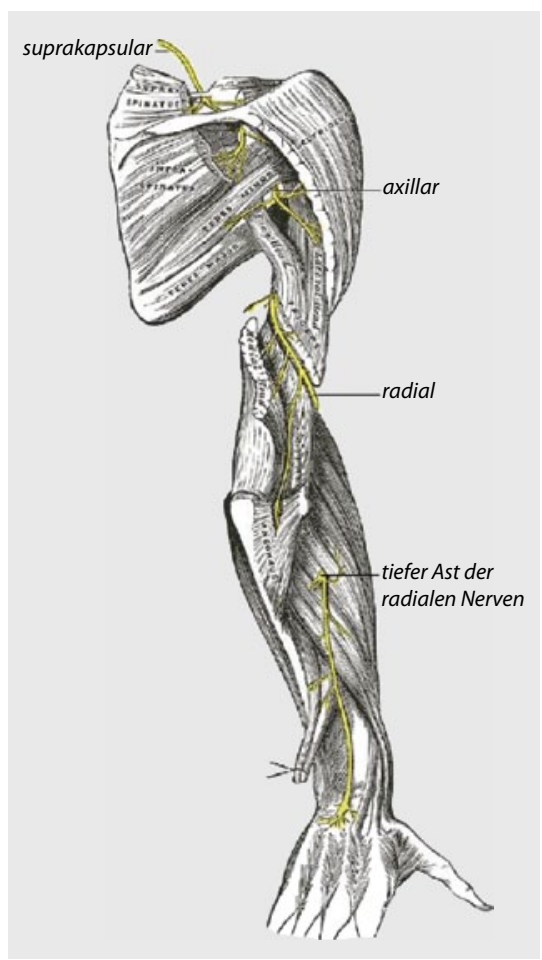

Abb. $1 \Delta$ Verlauf des N. radialis mit Abgang der Nervenäste. (Mod. nach [17])

bei ist eine komplette Durchtrennung des Nervs eher selten, sodass derzeit bei einer geschlossenen Fraktur und anschließend eingeschränkter Funktion des N. radialis zunächst ein abwartendes Vorgehen empfohlen wird, da es in über $85 \%$ der Fälle $\mathrm{zu}$ einer spontanen Regeneration kommt. Sollten jedoch erst nach der Reposition Symptome auftreten, besteht die Gefahr, dass der Nerv in die Fraktur eingeklemmt wurde. In diesen Fällen ist eine spontane Regeneration deutlich unwahrscheinlicher, sodass eine operative Exploration anzuraten ist. Aufgrund der hohen Rate an Radialislähmungen nach Oberarmfrakturen wird bei offenem Bruch die primäre Exploration mit Darstellung des Nervs dringend empfohlen, um eine langstreckige Unterbrechung, ein Einklemmen oder eine komplette Durchtrennung mit Dislokation der Enden durch eine Rekonstruktion, ggf. mit Nerveninterponat, z. B. durch einen Teil des N. suralis, primär behandeln zu können, wodurch sich die Chancen auf eine Wiederherstellung der Nervenfunktion deutlich verbessern.

Ist eine spontane Besserung der Nervenfunktion auch nach längerem Intervall oder nach dem Versuch einer Rekonstruktion nicht mehr zu erwarten, kommt ein operativer Sehnentransfer mit häufig gutem funktionellem Ergebnis in Frage.

\section{Ätiologie}

Ursachen für eine Läsion des N. radialis im Bereich des Oberarms sind in $\boldsymbol{\bullet}$ Tab. 1 aufgeführt. Hiervon zu unterscheiden sind Läsionen im Bereich des Plexus brachialis sowie eine distal gelegene Irritation z. B. im Rahmen eines Nervenengpasssyndroms.

\section{Anatomie}

Der N. radialis geht aus dem Fasciculus posterior des Plexus brachialis hervor und enthält Fasern aus C6-Th1. Er innerviert alle Streckermuskeln an Ober-, Unterarm und Hand. Begleitet von der A. profunda brachii verläuft er dorsal des Humerus im Sulcus nervi radialis, passiert das Septum intermusculare laterale und gelangt durch den Sulcus radialis (zwischen M. brachialis und brachioradialis) zur Ellenbeuge. Hier teilt er sich in einen tiefen motorischen Ast (N. interosseus posterior) und den sensiblen R. superficialis auf, der die Dorsalseite des distalen Unterarms und Daumenrückens versorgt.

In der Regel liegt folgende Reihenfolge der Nervenabgänge vor (• Abb. 1):

- Rr. articulares zum Schultergelenk, $\mathrm{N}$. cutaneus brachii posterior (dorsaler Oberarm) und N. cutaneus brachii lateralis (dorsolateraler Oberarm)

- M. triceps/M. anconeus

- BR

- ECRL

- N. cutaneus antebrachii posterior (Haut des Ellenbogens und des dorsalen Unterarms)

- M. supinator, ECRB

- EDC, ED quinti, APL, EPB, EIP, EPL

- Rr. articulares (Handgelenk, Grundgelenke D1-4)

\section{Inzidenz der Radialisparese}

Lokalisation und Frakturtyp sind entscheidende Parameter, um die Wahrscheinlichkeit für das Auftreten einer Radialisparese zu bestimmen.

Bei einer Fraktur des Humerus im Bereich des distalen Drittels ist eine Radialisparese mit 23,6\% als Begleitverletzung am wahrscheinlichsten, gefolgt von Frakturen in Schaftmitte, die zu 15,2\% mit einer Schädigung des N. radialis vergesellschaftet sind. Am seltensten kommt es im Bereich des proximalen Drittels mit einer Häufigkeit von 1,8\% zu einer Radialisparese [10].

Quer- und Spiralfrakturen führen besonders oft zu einer Radialisparese. Bei ungefähr 20\% dieser Verletzungen tritt hierbei eine Nervenfunktionsstörung auf. Die von Holstein u. Lewis [19] beschriebene distale extraartikuläre Spiralfraktur des Humerus mit Einklemmung des Nervs im Frakturspalt ist hierbei als für den N. radialis besonders gefährdend anzusehen. Hingegen ist das Risiko einer Radialisparese bei Schräg- oder Trümmerfrakturen des Humerus mit $8 \%$ bzw. $7 \%$ als signifikant geringer einzustufen $(p<0,001 ;[10$, 16]).

\section{Diagnostik}

An erster Stelle dienen Anamnese und klinische Untersuchung zur Feststellung des Lähmungsbildes. Sie können durch Elektrophysiologie (EMG der Kennmuskeln und Nervenleitgeschwindigkeit), Sonographie oder eine MRT ergänzt werden.

Die Wahrscheinlichkeit einer vollständigen Regeneration der Nervenfunktion hängt von der Form dessen Schädigung ab. Die leichteste Form (Neurapraxie), bei der weder die einzelne Nervenzelle noch die Hüllstrukturen in ihrer Kontinuität verletzt sind, hat das größte Regenerationspotenzial. Davon zu unterscheiden sind die unvollständige Nervendurchtrennung (Axonotmesis), bei der die Nervenhülle noch intakt ist, und die vollständige Nervendurchtrennung (Neurotmesis), bei der es zur kompletten Durchtrennung des Nervs und all seiner Strukturen kommt. Hierbei ist eine Restitutio ad integrum weniger wahrscheinlich, jedoch ebenfalls möglich.

Um eine exakte Aussage über die Regenerationswahrscheinlichkeit machen zu können, müsste also die Form der Schädigung bekannt sein. Dies ist jedoch im Allgemeinen mittels der klinischen und apparativen Diagnostik nicht möglich. 


\section{Anamnese}

Erhoben werden:

- Patientenmerkmale (Alter, Beruf, Einschränkungen, funktionelle Ziele),

- Zeitpunkt und Lokalisation der Nervenschädigung,

- Schädigungsmechanismus, z. B. Begleitverletzungen (Fraktur - Osteosynthese),

- bisherige Therapie (Nervenregeneration, -rekonstruktion) und Verlauf (Funktionswiederkehr) und

- bisherige Diagnostik (Röntgenbild der Fraktur, MRT, Sonographie, Elektrophysiologie).

\section{Klinische Untersuchung}

Ihr Ergebnis bestimmt das Lähmungsausmaß und die Möglichkeiten funktioneller Rekonstruktionen. Periphere Schädigungen des N. radialis führen je nach Höhe zum Verlust (• Tab. 2)

- der Ellenbogenstreckung,

- der Handgelenkstreckung in unterschiedlichem Ausmaß sowie

- der Extension der Fingergrundgelenke, des Daumens und seiner Abduktion.

Bei Läsionen im Schulterbereich kann der Ast des M. triceps betroffen sein und die Ellenbogenstreckung ausfallen.

Liegt die Läsion distal des Astes des M. triceps und proximal des muskulären Abgangs zum ECRL, fällt die Handgelenkstreckung komplett aus, es liegt eine komplette Fallhand vor.

Bei Schädigung auf Höhe oder distal des Ellenbogens ist der R. profundus nervi adialis (oder nervi interosseus posterior) betroffen. Dies führt zum Ausfall der Daumen- und Fingerstreckung in den MP-Gelenken. Die Handgelenkstreckung ist zwar geschwächt, kann aber durch den Erhalt des ECRL noch teilweise möglich sein, es handelt sich also um eine inkomplette Fallhand.

Bei proximaler sensibler Schädigung sind Ausfälle im Bereich der Haut über der Streckerseite des Oberarms zu verzeichnen, bei Läsion des R. superficialis besteht eine distale Gefühlsstörung des dorsalen Unterarms und der dorsa-

Trauma Berufskrankh 2012 - 14[Suppl 2]:197-202 DOI 10.1007/s10039-012-1856-y

(c) Springer-Verlag 2012

P.M. Vogt · M. Hiller

\section{N.-radialis-Ausfälle im Oberarmbereich. Wann muss wie reagiert werden?}

\section{Zusammenfassung}

Patienten mit einer Verletzung des N. radialis, die zum Symptomenkomplex der sog. Fallhand führen kann, leiden meist sehr und drängen oft auf eine rasche Operation. Der Zeitpunkt und die Technik der Versorgung hängen von verschiedensten Faktoren $a b$ und sollten individuell mit jedem Patienten besprochen und ein Therapieplan festgelegt werden. Dabei ist die Ursache der Verletzung unbedingt zu berücksichtigen. Die verletzungsbedingte Radialisparese stellt die häufigste traumatische Nervenläsion bei Fraktur der langen Röhrenknochen dar. Bei geschlossenem Bruch und anschließend eingeschränkter Funktion des N. radialis wird zunächst ein abwartendes Vorgehen empfohlen, da es in über $85 \%$ der Fälle zur sponta- nen Regeneration kommt. Aufgrund der hohen Rate an Radialislähmungen nach Oberarmfraktur wird bei offenem Oberarmbruch die primäre Exploration mit Darstellung des Nervs dringend empfohlen. Ist eine spontane Besserung der Nervenfunktion auch nach längerem Intervall oder nach dem Versuch einer Rekonstruktion nicht mehr zu erwarten, kommt ein operativer Sehnentransfer mit häufig gutem funktionellem Ergebnis in Frage.

\section{Schlüsselwörter}

Oberarmfraktur · Verletzung peripherer Nerven - Radialisparese - Nervenregeneration . Operative Versorgung

\section{Radial nerve paralysis in the upper arm. When should one react and how?}

\section{Abstract}

Patients with injury to the radial nerve which can lead to the symptom complex known as wrist drop or carpoptosis, mostly suffer a great deal and often insist on an urgent operation. The timing and technique for treatment depend on various factors and should be discussed individually with each patient and a therapy plan should be determined. In this context the cause of the injury is of absolute importance. Paralysis of the radial nerve due to injury represents the most common traumatic neural lesion in fractures of the long bones. For closed fractures and subsequent limited function of the radial nerve, a waiting procedure is initially recommended

len Grund- und Mittelglieder der radialen 2 1/2 Finger.

\section{Therapie}

\section{Art}

Liegt die Nervenläsion bei einer peripheren Lähmung mit Schädigung des unteren Motoneurons weniger als 12 Monate zurück, sollte immer eine nervale Rekonstruktion versucht werden (entweder durch Nervennaht, Neurolyse, Nerventransplantation oder -transposition), da because spontaneous regeneration occurs in more than $85 \%$ of cases. For open fractures primary exploration with exposure of the nerve is urgently recommended because of the high rate of paralysis of the radial nerve. If spontaneous improvement in nerve function can no longer be expected after a long time interval or reconstruction attempts, surgical transfer of tendons can be performed often with good functional results.

\section{Keywords}

Humeral fractures - Peripheral nerve injuries . Radial nerve paralysis - Nerve regeneration . Surgical treatment 


\begin{tabular}{|c|c|c|c|}
\hline $\begin{array}{l}\text { Ausmaß der } \\
\text { Läsion }\end{array}$ & $\begin{array}{l}\text { Höhe der Nerven- } \\
\text { schädigung }\end{array}$ & $\begin{array}{l}\text { Muskel-/Hautinnerva- } \\
\text { tion }\end{array}$ & Funktionsverlust \\
\hline $\begin{array}{l}\text { Hoch proximal- } \\
\text { komplette } \\
\text { Radialisparese }\end{array}$ & $\begin{array}{l}\text { Läsion proximal des } \\
\text { Astes des M. triceps }\end{array}$ & M. triceps & $\begin{array}{l}\text { Keine Ellenbogen- } \\
\text { streckung, keine Hand- } \\
\text { gelenk-, Finger- und } \\
\text { Daumenstreckung }\end{array}$ \\
\hline $\begin{array}{l}\text { Proximal - } \\
\text { komplette Fall- } \\
\text { hand }\end{array}$ & $\begin{array}{l}\text { Lähmung } \\
\text { proximal des ECRB- } \\
\text { Astes }\end{array}$ & $\begin{array}{l}\text { BR } \\
\text { ECRL }\end{array}$ & $\begin{array}{l}\text { Keine Streckung von } \\
\text { Handgelenk, Fingern } \\
\text { und Daumen }\end{array}$ \\
\hline R. superficialis & & $\begin{array}{l}\text { Radialseite Handrücken } \\
\text { Streckseite Daumen } \\
\text { Grundglied Zeige- und } \\
\text { Mittelfinger }\end{array}$ & \\
\hline $\begin{array}{l}\text { Distal: R. profundus } \\
\text { (nervi interosseus } \\
\text { posterior) - } \\
\text { inkomplette } \\
\text { Fallhand }\end{array}$ & $\begin{array}{l}\text { Lähmung distal des } \\
\text { ECRL-Astes }\end{array}$ & $\begin{array}{l}\text { ECRB } \\
\text { M. supinator } \\
\text { EDC } \\
\text { EDM } \\
\text { ECU } \\
\text { APL } \\
\text { EPB/EPL/EIP }\end{array}$ & $\begin{array}{l}\text { Keine Streckung von } \\
\text { Fingern und Daumen, } \\
\text { Radialdeviation des } \\
\text { Handgelenks bei } \\
\text { Extension }\end{array}$ \\
\hline $\begin{array}{l}\text { Distaler N. interos- } \\
\text { seus posterior }\end{array}$ & & $\begin{array}{l}\text { Periost von Ulna, Radius, } \\
\text { Hand- und Fingerknochen }\end{array}$ & $\begin{array}{l}\text { Reduzierte Schmerz- } \\
\text { empfindung }\end{array}$ \\
\hline \multicolumn{4}{|c|}{$\begin{array}{l}\text { APL M. abductor pollicis longus, BR M. brachioradialis, ECRB M. extensor carpi radialis brevis, ECRL M. extensor } \\
\text { carpi radialis longus, ECU M. extensor carpi ulnaris, EDC M. extensor digitorum communis, EDM M. extensor } \\
\text { digiti minimi, EIP M. extensor indicis proprius, EPB M. extensor pollicis brevis, EPL M. extensor pollicis longus }\end{array}$} \\
\hline
\end{tabular}

\begin{tabular}{|c|c|c|}
\hline 1 & \multicolumn{2}{|c|}{ Keine spontane Funktionsrückkehr (durch Nervenregeneration) zu erwarten } \\
\hline \multirow[t]{5}{*}{2} & \multirow{5}{*}{$\begin{array}{l}\text { Operative } \\
\text { Alternativen } \\
\text { ausgeschöpft }\end{array}$} & Nervennaht oder -dekompression \\
\hline & & Primärrekonstruktion von Nerven, Sehnen und Muskeln \\
\hline & & Nerventransposition mit kurzer Regenerationsstrecke \\
\hline & & Tenotomie/Sehnenverlängerung, um Gelenkbeweglichkeit zu verbessern \\
\hline & & Tenodese (Bewegung des proximalen Gelenks bewirkt Bewegung) \\
\hline 3 & \multicolumn{2}{|c|}{ Keine weitere Verbesserung durch Handtherapie } \\
\hline 4 & \multicolumn{2}{|c|}{ Einsichtsfähigkeit und Motivation (v. a. zur Nachbehandlung) des Patienten } \\
\hline 5 & \multicolumn{2}{|c|}{ Behandlungsplan nach körperlicher Untersuchung und Beratung des Patienten } \\
\hline 6 & \multicolumn{2}{|c|}{ Verzichtbare und geeignete Spendermuskeln (Kraftgrad $\geq \mathrm{M} 4$ ) vorhanden } \\
\hline 7 & \multicolumn{2}{|c|}{ Günstige Weichteilsituation } \\
\hline 8 & \multicolumn{2}{|c|}{ Möglichst freie passive Gelenkbeweglichkeit } \\
\hline
\end{tabular}

chirurgischen funktionellen Muskeltransplantation.

Solange eine Funktionsrückkehr nach Nervenverletzung noch realistisch ist (etwa 12 bis 18 Monate nach der Läsion), sollten zunächst eine Nervenrekonstruktion oder -transposition versucht werden.

\section{Konservative Therapie}

Ziele der nichtoperativen Behandlung sind:

- Erhalt der freien Gelenkbeweglichkeit und

- Vermeidung von Kontrakturen (auch der 1. Kommissur), idealerweise unter handtherapeutischer Aufsicht

(KG, Ergotherapie).

Der Patient muss von Anfang an die Verantwortung zur selbstständigen Eigenbeübung übernehmen.

Spezielle Radialisschienen beinhalten meist ein Zugsystem zur passiven Streckung von Fingern und Daumen, z. B. durch Gummizügel, die aktive Flexion ist frei möglich. Es empfiehlt sich eine Nachtlagerungsschiene in Streckstellung, um einer Verkürzung der antagonistischen Flexoren und einem Muskelungleichgewicht nach der erfolgreichen Nervenregeneration vorzubeugen.

\section{Operative Therapie}

\section{Nervenrekonstruktion}

Direkte Nervennaht. Bei ihr werden bei teilweiser oder kompletter Durchtrennung des Nervs dessen Scheiden unter mikroskopischer Sicht readaptiert. Hiernach kommt es zu einem Aussprossen des Nervs nach distal entlang der rekonstruierten Nervenscheide als Leitstruktur. Hierdurch kann u. U. die Muskelfunktion komplett wiederhergestellt werden. Wichtig ist hierbei, dass das Intervall bis zum Erreichen der motorischen Endplatte durch das nachwachsende Axon nicht unbegrenzt ist, da es nach etwa 12 bis 18 Monaten zu deren Untergang kommen kann.

Interposition. Hier kommt u. a. der N. suralis in Frage.

Neurotisation. Als Beispiel sei die Neuotisation des $\mathrm{N}$. interosseus posterior durch (redundante) Äste des N. medianus zu FDS, PL, FCR genannt.

\section{Dekompression des Nervs bei Nervenkompression}

Motorische Ersatzoperation. Bei ihr werden irreparabel verlorene Muskelfunktionen durch eine Transposition gesunder Sehnen-Muskel-Einheiten ersetzt und so die Funktion gelähmter oder zerstörter Muskelgruppen wiederhergestellt.

Motorische Ersatzoperationen werden in der Regel als Sekundäroperationen durchgeführt, wenn klinische und elektrophysiologische Zeichen der Reinnervation des gelähmten Muskels nach etwa 6 Monaten ausbleiben. Als Primäreingriffe dienen sie dazu, Funktionsausfälle bis zur Muskelreinnervation temporär zu kompensieren. Denn bei weit proximaler Nervenschädigung und einer durchschnittlichen Regenerationsgeschwindigkeit von 1-2 mm/Tag erreichen die auswachsenden Axone die periphere Muskulatur oft erst nach Monaten. Bei temporären Ersatzoperationen werden die Empfängersehnen daher nicht durchtrennt, sondern es wird eine Seit-zu-SeitNaht vorgenommen, damit die Operation bei ausreichender natürlicher Reinnervation reversibel bleibt. Theoretisch bleiben 


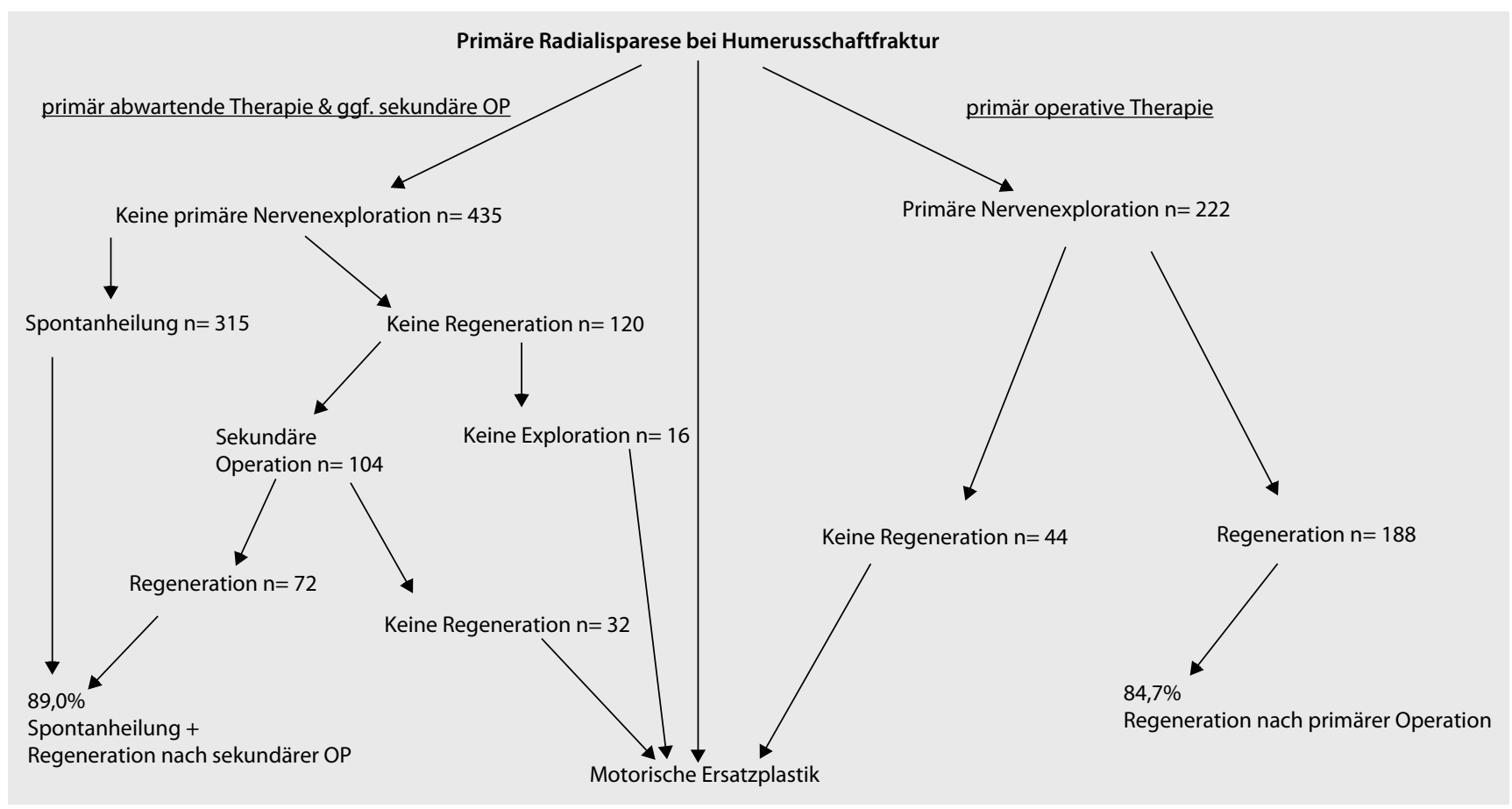

Abb. $2 \Delta$ Regenerationsrate bei primärer Radialisparese in Abhängigkeit von der Therapiestrategie, OP Operation. (Mod. nach [33])

Muskelersatzoperationen auch viele Jahre nach der Lähmung möglich.

Allgemeine Voraussetzungen für motorische Ersatzoperationen sind in - Tab. 3 dargestellt. Ein Fehlen einer dieser Faktoren stellt in der Regel eine Kontraindikation zur Muskelersatzoperation dar oder erfordert präoperativ entsprechende Gegenmaßnahmen.

Zur Wiederherstellung der Radialisfunktion existiert eine große Zahl möglicher Sehnentranspositionen. Allein Boyes [4] stellte bereits 1960 insgesamt 58 unterschiedliche Optionen dar. Als Beispiel wird im Folgenden die Technik nach Merle d'Aubigné dargestellt.

Technik nach Merle d' Aubigné. Die Handgelenkextension wird durch die Umlagerung der PT-Sehne auf die Sehne von ECRL und ECRB wiederhergestellt. Eine Fingerextension ist durch die Transposition der Sehne des FCU zum EDC und EPL zu erreichen, und die Extension des Daumens wird durch eine Verlagerung der Sehne des PL auf die des EPB und APL gewährleistet [22].

Nach Freigabe der Belastung kann die Hand nach weiteren 6 bis 8 Wochen wieder gut im Alltag eingesetzt werden. Bei einwandfreier chirurgischer Technik und aktiver Kooperation des Patienten bei der Nachbehandlung hat die Radialisersatzplastik eine der besten Erfolgsaussichten aller sekundären funktionellen Rekonstruktionsverfahren an den Extremitäten. Eine Wiederaufnahme der vollen Aktivität am Arbeitsplatz und in der Freizeit ist nicht selten bereits nach einigen Monaten möglich.

\section{Zeitpunkt der Therapie}

Green [18] schlug bei geschlossenen $\mathrm{Hu}-$ merusschaftfrakturen ein eher zurückhaltendes Vorgehen vor und ggf. im Verlauf nach ausbleibender Spontanregeneration eine sekundäre Exploration mit ggf. operativer Rekonstruktion. Dieses Vorgehen deckt sich mit den Ergebnissen, die Shao et al. [33] beschrieben und die belegen, dass kein signifikanter Unterschied im Outcome besteht, was den Vergleich zwischen primär abwartender Therapie mit ggf. sekundärer Operation und einer primär operativen Therapie bei geschlossenen Oberarmschaftfrakturen betrifft ([18, 33], Abb. 2).

Selbst hinsichtlich des Zeitpunktes der sekundären Exploration besteht in der Literatur Uneinigkeit. Shaw u. Sakellarides [34] schlugen eine Exploration 7 bis
8 Wochen nach dem Trauma vor, sollte bis dahin kein Anhalt für eine beginnende Regeneration bestehen. Als Grund hierfür wurden folgende Argumente angeführt: Bei allen den von ihnen untersuchten $\mathrm{Pa}$ tienten waren Zeichen einer Rückkehr der Nervenfunktion innerhalb der ersten 2 Monate nachweisbar. Die Knochenheilung war in der Regel nach dieser Zeit abgeschlossen. Außerdem könne hierdurch eine unnötige Operation bei spontaner Heilung vermieden werden. Weiter sei diese Zeit kurz genug, dass es noch zu keiner Retraktion der Nervenenden, aber bereits zur Ausbildung von Neuromen gekommen sei, sodass diese adäquat reseziert werden können [18].

Green [18] schlug dagegen vor, das Intervall bis zur sekundären Exploration entsprechend der zu erwartenden Regenerationsdauer gemäß $\bullet$ Abb. 3 nach Seddon $[29,30,31]$ zu wählen.

\section{Fazit für die Praxis}

Als häufigste Ursache einer Radialisparese mit der Folge einer kompletten Fallhand ist die Oberarmschaftfraktur anzusehen. Eine Radialisparese tritt nach $\mathrm{Hu}$ merusschaftfraktur in etwa $12 \%$ der Fäl- 


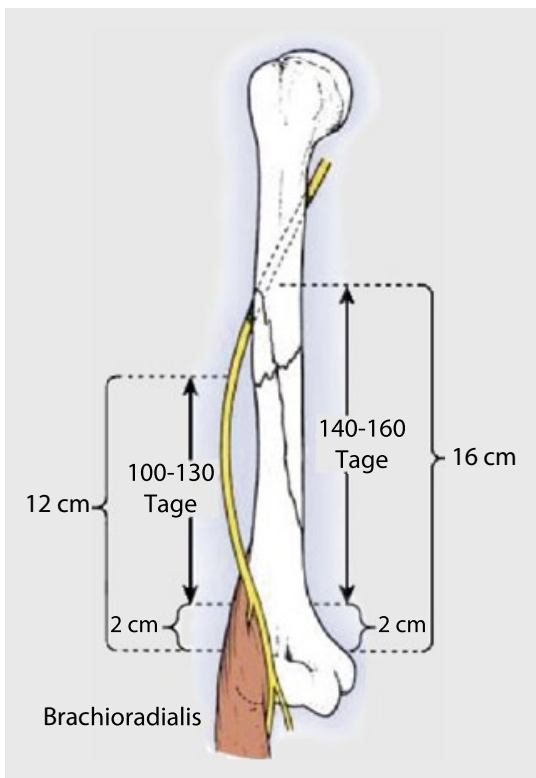

Abb. $3 \Delta$ Berechnung der Regenerationszeit, die verstreicht, bevor Zeichen der Regeneration des $\mathrm{N}$. radialis nach Oberarmschaftfrakturen, welche dessen Läsion auslösten, zu erwarten sind, links Angaben für Querfraktur, rechts Angaben für Schrägfraktur des Humerusschafts. (Mod. nach [31, 37], mit freundl. Genehmigung von John Wiley \& Sons)

le auf. Dabei ist eine Nervenschädigung am häufigsten bei Schräg- und Spiralfrakturen im mittleren und insbesondere im distalen Drittel aufgrund des Verlaufs des Nervs um den Knochen herum zu erwarten.

Unabhängig davon, ob eine primär abwartende Strategie mit ggf. in der Folge sekundärer Operation oder die primäre Operation gewählt wird, kommt es in über $84 \%$ der Fälle zur Regeneration der Nervenfunktion. Aufgrund dieser hohen Spontanheilungsrate sollte von einer primär operativen Exploration abgesehen werden, jedoch sollte diese bei offenen Frakturen bzw. offenen Stich- oder Schnittverletzungen, Hinweis auf eine relevante Nervenverletzung mit Unterbrechung des $\mathrm{N}$. radialis oder sekundärer Funktionsstörung nach Reposition frühzeitig durchgeführt werden. Bei persistierender Radialislähmung über ein längeres Intervall, entsprechend der Regenerationszeit nach Seddon $([29,30,31]$,

- Abb. 3), seit der Verletzung empfehlen wir ebenfalls die Exploration. Liegt die Nervenläsion weniger als 6 Monate zurück, sollte immer eine nervale Rekonstruktion versucht werden - entweder durch direkte Nervennaht, Nerveninterposition (z. B. N. suralis) oder die Neurotisation -, da eine anatomische Muskelreinnervation in der Regel die besten Ergebnisse erbringt. Sollte eine Reinnervation der gelähmten Muskulatur nicht möglich sein, kommt eine motorische Ersatzoperation durch Sehnentransfer in Frage.

\section{Korrespondenzadresse}

Prof. Dr. P.M. Vogt

Klinik und Poliklinik für Plastische, Hand- und Wiederherstellungschirurgie, Medizinische Hochschule Hannover, Carl-Neuberg-Straße 1 1, 30625 Hannover vogt.peter@mh-hannover.de

Interessenkonflikt. Der korrespondierende Autor gibt an, dass kein Interessenkonflikt besteht.

\section{Literatur}

1. Bevin AG (1976) Early tendon transfer for radial nerve transection. Hand 8:134

2. Biesalski K, Mayer L (1916) Die physiologische Sehnenverpflanzung. Springer, Berlin Heidelberg New York

3. Bleeker WA, Nijsten MW, Ten Duis HJ (1991) Treatment of humeral shaft fractures related to associated injuries. A retrospective study of 237 patients. Acta Orthop Scand 62:148-153

4. Boyes JH (1960) Tendon transfers for radial palsy. Bull Hosp Jt Dis 21:97

5. Brand PW, Hollister AM (1999) Clinical mechanics of the hand, 3. Aufl. Mosby, St. Louis

6. Brüser $P$ (1999) Motorische Ersatzoperationen an der oberen Extremität. Dtsch Arztebl 96A:1134-1139

7. Buck-Gramcko D (1973) Erfahrungen mit Radialis Ersatzoperationen. Handchirurgie 5:105

8. Chuinard RG, Boyes JH, Stark HH, Ashworth CR (1978) Tendon transfers for radial nerve palsy: use of superficialis tendons for digital extension. J Hand Surg [Am] 3(6):560-570

9. Dabas V, Suri T, Surapuraju PK et al (2011) Functional restoration after early tendon transfer in high radial paralysis. J Hand Surg Eur 36(2):135-140

10. Daecke W, Geiger F (2008) Radialisparese - gesicherte Literaturergebnisse. Trauma Berufskrankh [Suppl 2] 10:267-271

11. Franke $F$ (1898) Über die operative Behandlung der Radialislähmung nebst Bemerkungen über die Sehnenüberpflanzungen bei spastischen Lähmungen. Arch Klin Chir 57:763

12. Friden J (2005) Neue Konzepte zur Rekonstruktion der Arm- und Handfunktion bei Tetraplegie - Grundlagenforschung und klinische Anwendung. Handchir Mikrochir Plast Chir 37:223-229

13. Friden J (Hrsg) (2005) Tendon transfers in reconstructive hand surgery. Taylor \& Francis, London

14. Gohritz A, Vogt PM (2011) Motorische und nervale Ersatzoperationen bei Lähmungen an Unterarm und Hand. In: Vogt PM (Hrsg) Praxis der Plastischen Chirurgie. Springer, Berlin Heidelberg New York, S 367378
15. Gohritz A, Friden J, Herold C et al (2007) Ersatzoperationen bei Ausfall motorischer Funktionen an der Hand. Unfallchirurg 110:759-776

16. Gousheh J, Arasteh E (2006) Transfer of a single flexor carpi ulnaris tendon for treatment of radial nerve palsy. J Hand Surg [Br] 31(5):542-546

17. Gray H (1918) Anatomy of the human body. Ley \& Febiger, Philadelphia

18. Green DP (2011) Radial nerve palsy. In: Green DP, Wolfe SW, Hotchkiss RN et al (Hrsg) Operative hand surgery, 6. Aufl. Churchill Livingstone, Edinburgh London New York

19. Holstein A, Lewis GM (1963) Fractures of the humerus with radial-nerve paralysis. J Bone Joint Surg Am 45:1382-1388

20. Krishnan KG, Schackert G (2008) An analysis of results after selective tendon transfers through the interosseous membrane to provide selective finger and thumb extension in chronic irreparable radial nerve lesions. J Hand Surg [Am] 33(2):223-231

21. Kruft S, Heimburg D von, Reill P (1997) Treatment of irreversible lesion of the radial nerve by tendon transfer: indication and long-term results of the Merle d'Aubigné procedure. Plast Reconstr Surg 100(3):610-616

22. Merle d'Aubigné R, Lange $P$ (1946) Transplantations tendineuses dans le traitement des paralysies radiales post-traumatiques. Sem Hop Paris 22:1666-1680

23. Nigst H (1990) Ersatzoperationen bei Radialislähmungen. In: Buck-Gramcko D, Nigst H (Hrsg) Motorische Ersatzoperationen der oberen Extremität, Bd. 2: Hand und Unterarm. Hippokrates, Stuttgart, S 18-53

24. Perthes G (1918) Über Sehnenoperationen bei irreparabler Radialislähmung nebst Studien über die Sehnenverpflanzung und Tenodese im allgemeinen. Bruns Beitr Klin Chir 113:289

25. Richards RR (2003) Tendon transfers for failed nerve reconstruction. Clin Plast Surg 30:223-245

26. Riordan DC (1974) Radial nerve paralysis. Orthop Clin North Am 5:283-287

27. Ropars M, Dréano T, Siret $P$ et al (2006) Long-term results of tendon transfers in radial and posterior interosseous nerve paralysis. J Hand Surg [Br] 31(5):502506

28. Rudigier J (1991) Ersatzoperationen an Oberarm und Ellbogen. In: Buck-Gramcko D, Nigst H (Hrsg) Bibliothek für Handchirurgie. Motorische Ersatzoperationen der oberen Extremität. Bd 1: Schultergürtel, Oberam und Ellbogen. Hippokrates, Stuttgart

29. Seddon $H$ (1972) Factors influencing indications for operation. In: Seddon $\mathrm{H}$ (Hrsg) Surgical disorders of the peripheral nerves. Williams \& Wilkins, Baltimore, S 240-245

30. Seddon $H$ (1947) Nerve lesions complicating certain closed bone injuries. JAMA 135:691-694

31. Seddon H (1975) Surgical disorders of the peripheral nerves. Churchill Livingstone, Edinburgh London New York

32. Shah J, Bhatti NA (1983) Radial nerve paralysis associated with fractures of the humerus. Clin Orthop Relat Res 172:171-176

33. Shao YC, Harwood P, Grotz MR et al (2005) Radial nerve palsy associated with fractures of the shaft of the humerus: a systematic review. J Bone Joint Surg Br 87:1647-1652

34. Shaw J, Sakellarides H (1967) Radial-nerve paralysis associated with fractures of the humerus: a review of forty-five cases. J Bone Joint Surg Am 49:899-902

35. Sudeck P (1943) Gedanken zur Plastik bei Radialislähmung. Chirurgie 15:665

36. Switlick DN, Sheppard JE (2005) Tendon transfers for peripheral nerve injuries. In: Trumble T, Cornwall R, Budoff JE (Hrsg) Hand, shoulder \& shoulder: core knowledge in orthopaedics. Mosby, Philadelphia

37. Zachary R (1946) Tendon transplantation for radial paralysis. Br J Surg 1946; 23:358-364 